\title{
Targeting apoptosis in the control of inflammation
}

\author{
H-U. Simon
}

\begin{abstract}
Apoptosis is the most common form of physiological cell death and serves to eliminate cells that are genomically altered and therefore potentially harmful to the body, senescent or become replaced by functionally different cell types. It is defined by morphological criteria, including chromatin condensation, nuclear fragmentation, plasma membrane blebbing, organelle disruption, loss of adhesion and rounding (in adherent cells), and cell shrinkage. Apoptosis is required for normal development and maintenance of tissue homeostasis, and its dysregulation is associated with various pathological conditions. For instance, there is often too little apoptosis in cancer and there is usually too much apoptosis in neurodegeneration and acquired immune deficiency syndrome. In inflammatory responses, delayed apoptosis of inflammatory cells contributes to their accumulation in order to efficiently eliminate the pathogen. This article provides a short overview regarding dysregulated apoptosis associated with inflammation.
\end{abstract}

\section{Bacterial and fungal infection}

Delayed neutrophil apoptosis has been associated with several acute and chronic inflammatory diseases (e.g. pneumonia, sepsis, cystic fibrosis) and appears to be largely mediated by excessive production of granulocyte colony-stimulating factor and granulocyte/macrophage colony-stimulating factor (GMCSF) [1]. The induction of neutrophil apoptosis during the resolution of a neutrophilic inflammatory response can be mimicked in vitro by culturing the cells in the absence of sufficient concentrations of survival factors, a process called spontaneous apoptosis. Most studies have been performed on purified blood neutrophils, aiming to understand the molecular events that control apoptosis in these cells. Tyrosine kinases, the phosphoinositide 3-kinase components of the mitogen-activated protein kinase pathways, the cyclic adenosine monophosphate/ protein kinase A pathway, and protein kinase $\mathrm{C}$ pathways play a role [2]. An early antiapoptotic effect is the phosphorylation of $\mathrm{Bad}$, which leads to its translocation into the cytosol and its inability to interact with antiapoptotic Bcl-2 family members [3]. Later on, further antiapoptotic effects occur by modulation of the expression of apoptosis-regulating molecules of the Bcl-2 and inhibitor of apoptosis protein (IAP) families.

Mechanisms that control the accumulation of neutrophils at sites of inflammation most likely involve all events that limit the synthesis of neutrophil survival factors in inflammatory and structural cells. Intracellular inhibitors of cytokine signalling expressed in neutrophils are the Src homology domain 2 (SH2)-containing tyrosine phosphatase-1 (SHP-1) [4] and cytokine-inducible SH2-containing protein-1 [5]. Evidence for a functional role of SHP-1 as an inhibitory phosphatase activated by death receptors has been obtained

Correspondence: H-U. Simon, Dept of Pharmacology, University of Bern, Friedbühlstrasse 49, CH-3010 Bern, Switzerland. Fax: 41 316324992. E-mail: hus@pki.unibe.ch by comparing neutrophils from normal and SHP-1-deficient mice. Inhibition of GM-CSF-mediated survival by simultaneous activation of Fas receptors was significantly reduced in SHP-1-deficient neutrophils, indicating that SHP-1 is important in limiting antiapoptotic signals. Such a mechanism could largely explain why SHP-1-deficient mice demonstrate persistent neutrophilic inflammation [4]. Further support for a role of SHP-1 in regulating neutrophil numbers was obtained by the observation that this phosphatase is overexpressed in patients with severe neutropaenia. Increased enzymatic SHP-1 may block cytokine effects that are important for both antiapoptosis and differentiation, resulting in low neutrophil numbers [6]

\section{Allergic inflammation}

Eosinophils are prominent effector cells in many chronic allergic and parasitic inflammatory responses. Like other leukocytes, they are constantly generated in the bone marrow. To maintain cellular homeostasis, aged eosinophils must die by apoptosis and be removed in the absence of inflammation [7]. Interleukin (IL)-5 appears to be a specific survival factor for eosinophils, at least within the human system. Therefore, and not surprisingly, eosinophilia and high IL-5 expression levels have often been associated, especially in chronic allergic disorders such as bronchial asthma and atopic dermatitis. The phenomenon of delayed eosinophil apoptosis has been demonstrated in nasal polyp explants [8] as well as in purified blood eosinophils from patients with atopic dermatitis [9] and some patients with the hypereosinophilic syndrome [10]. Moreover, it appears that the severity of asthma negatively correlates with the amount of eosinophil apoptosis in the airways [11]. These findings suggest delayed eosinophil apoptosis as a hallmark of tissue eosinophilia.

Besides eosinophils, T-cells also have an increased life span at the site of allergic inflammation. IL-2, -4 and -15 are important survival factors that prevent apoptosis even in activated and Fas-sensitive T-cells [12]. Both T-cells and eosinophils are believed to induce apoptosis in epithelial cells by different mechanisms, and the resulting epithelial damage may largely contribute to the symptoms seen in asthma [13] and atopic dermatitis [14].

\section{Autoimmune inflammation}

Rheumatoid arthritis (RA) is a common autoimmune disease in which patients suffer from chronic inflammatory synovitis that is dominated by the presence of macrophages, neutrophils, lymphocytes and synovial fibroblasts. Synovial macrophages are highly resistant to apoptotic stimuli [15]. Tumour necrosis factor (TNF)- $\alpha$ stimulation appears to participate in this antiapoptotic effect, since it results in increased expression of FLICE-like inhibitory protein (FLIP), 
the Bcl-2 family member A1, and the Bcl-2 family member Mcl-1 in these cells. Therefore, anti-TNF- $\alpha$ therapies may partially act via the promotion of macrophage apoptosis. Synovial fibroblasts have also been identified as important contributors to the pathogenesis of RA and apoptosis of these cells is rare. The relative insensitivity of synovial fibroblasts to undergo apoptosis may be the consequence of high expression of FLIP and X-linked IAP, as well as inactivation of p53. Moreover, it has been shown that synovial fluid from RA patients delays neutrophil apoptosis, and interferon- $\beta$ and adenosine have been identified as the key mediators responsible for this effect. Although defective Fas ligand (FasL) expression on activated synovial $\mathrm{T}$-cells has been reported, this finding may not be of pathogenic relevance since treatment with depleting monoclonal antibodies directed against T-cells has not been effective.

Patients with systemic lupus erythematosus have increased levels of soluble Fas, which competitively inhibits the FasLFas interactions. The resulting decrease in Fas-mediated apoptosis may contribute to the accumulation of autoimmune cells in this disorder. In patients with leukopaenia or thrombopaenia, autoantibodies that induce massive apoptosis (often in conjunction with the complement system) may cause the disease. In other circumstances, the expression of growth and differentiation factors may not be sufficient to prevent apoptosis of haematopoietic precursor cells. In autoimmune endocrine diseases, such as type-1 diabetes, $\beta$-cells of islets of Langerhans in the pancreas undergo increased apoptosis due to attacks of autoreactive FasLexpressing CD4+ and CD8+ T-cells. There are many more autoimmune diseases for which published reports on deregulated apoptosis exist.

One example is the autoimmune lymphoproliferative syndrome (ALPS) in which dominant mutations in the Fas receptor, FasL or other genes in the Fas pathway are found [16]. ALPS is clinically characterised by massive lymphadenopathy and splenomegaly, which typically presents in childhood. These symptoms are often accompanied by autoimmune haemolytic anaemia, thrombocytopaenia and other autoimmune manifestations. In addition, patients have markedly increased numbers of circulating double-negative T-cells (CD4CD8-). ALPS patients also have increased numbers of circulating B-cells and polyclonal hypergammaglobulinaemia.

\section{Conclusion}

Normal cell turnover through properly regulated apoptosis is particularly important to maintain the function of the immune system and other complex physiological processes in higher organisms. A study of the molecular basis of many diseases reveals an apoptotic component that either accounts for or contributes to disease progression. Growing knowledge on the apoptotic machinery together with technological developments and advances in combinatorial chemistry and gene therapy has paved the way for the more effective treatment of diseases associated with deregulated apoptosis.

\section{References}

1. Dibbert B, Weber M, Nikolaizik WH, et al. Cytokinemediated Bax deficiency and consequent delayed neutrophil apoptosis: A general mechanism to accumulate effector cells in inflammation. Proc Natl Acad Sci USA 1999; 96: 13330-13335.

2. Simon HU. Neutrophil apoptosis pathways and their modifications in inflammation. Immunol Rev 2003; 193: 101-110.

3. Downward J. How BAD phosphorylation is good for survival. Nat Cell Biol 1999; 1: E33-E35.

4. Daigle I, Yousefi S, Colonna M, Green DR, Simon HU. Death receptors bind SHP-1 and block cytokine-induced anti-apoptotic signaling in neutrophils. Nat Med 2002; 8: 61-67.

5. Yousefi S, Cooper PR, Mueck B, Potter SL, Jarai G. cDNA representational difference analysis of human neutrophils stimulated by GM-CSF. Biochem Biophys Res Commun 2000; 277: 401-409.

6. Tidow N, Kasper B, Welte K. SH2-containing protein tyrosine phosphatases SHP-1 and SHP-2 are dramatically increased at the protein level in neutrophils from patients with severe congenital neutropenia (Kostmann's syndrome). Exp Hematol 1999; 27: 1038-1045.

7. Simon HU. Eosinophil apoptosis - pathophysiologic and therapeutic implications. Allergy 2000; 55: 910-915.

8. Simon HU, Yousefi S, Schranz C, Schapowal A, Bachert C, Blaser K. Direct demonstration of delayed eosinophil apoptosis as a mechanism causing tissue eosinophilia. J Immunol 1997; 158: 3902-3908.

9. Wedi B, Raap U, Lewrick H, Kapp A. Delayed eosinophil programmed cell death in vitro: a common feature of inhalant allergy and extrinsic and intrinsic dermatitis. J Allergy Clin Immunol 1997; 100: 536-543.

10. Plötz SG, Dibbert B, Abeck D, Ring J, Simon HU. Bcl-2 expression by eosinophils in a patient with hypereosinophilia. J Allergy Clin Immunol 1998; 102: 1037-1040.

11. Vignola AM, Chanez P, Chiappara G, et al. Evaluation of apoptosis of eosinophils, macrophages, and T-lymphocytes in mucosal biopsy specimens of patients with asthma and chronic bronchitis. J Allergy Clin Immunol 1999; 103: 563573.

12. Akdis CA, Akdis M, Trautmann A, Blaser K. Immune regulation in atopic dermatitis. Curr Opin Immunol 2000; 12 : 641-646.

13. Trautmann A, Schmid-Grendelmeier $\mathrm{P}$, Kruger $\mathrm{K}$, et al. T-cells and eosinophils cooperate in the induction of bronchial epithelial cell apoptosis in asthma. J Allergy Clin Immunol 2002; 109: 329-337.

14. Trautmann A, Akdis M, Schmid-Grendelmeier $\mathrm{P}$, et al. Targeting keratinocyte apoptosis in the treatment of atopic dermatitis and allergic contact dermatitis. J Allergy Clin Immunol 2001; 108: 839-846.

15. Pope RM. Apoptosis as a therapeutic tool in rheumatoid arthritis. Nat Rev 2002; 2: 527-535.

16. Siegel RM, Chan FKM, Chun HJ, Lenardo MJ. The multifaceted role of Fas signaling in immune cell homeostasis and autoimmunity. Nat Immunol 2000; 1: 469-474. 